\title{
Creencias matemáticas profesadas e implícitas de profesores universitarios de matemáticas
}

\section{Professed and implicit mathematical beliefs of university mathematics teachers}

\author{
Antonia Hernández Moreno ${ }^{1}$ \\ Yuridia Arellano García ${ }^{2}$ \\ Gustavo Martínez Sierra ${ }^{3}$
}

\begin{abstract}
Resumen: Las investigaciones de creencias de profesores se han centrado en el nivel básico y en el de formación, con una gran tendencia al uso de las escalas Likert. Por otro lado, investigaciones sobre emociones de profesores desde las teorías de valoración, sostienen que esas valoraciones son realizadas en función de las creencias, metas, etc. de los profesores. El objetivo de nuestra investigación fue utilizar esta relación entre emoción-creencia para identificar, además de creencias profesadas, creencias implícitas en las valoraciones cognitivas reportadas en auto-informes diarios sobre las experiencias de clase de dos profesores de nivel superior. Identificamos dos tipos de creencias implícitas: referidas al aprendizaje y a la gestión en el aula. Además, 22 creencias matemáticas profesadas por los profesores que organizamos en cinco categorías: matemáticas es, aprender matemáticas es, para aprender matemáticas se debe, enseñar matemáticas es, y para enseñar matemáticas se debe. Presentamos
\end{abstract}

Fecha de recepción: 25 de marzo de 2019. Fecha de aceptación: 29 de mayo de 2020

${ }^{1}$ Centro de Investigación en Matemática Educativa, Facultad de Matemáticas, Universidad Autónoma de Guerrero, antonia.inves@gmail.com, orcid.org/0000-0001-9897-7626

2 Centro de Investigación en Matemática Educativa, Facultad de Matemáticas, Universidad Autónoma de Guerrero, yaregar@gmail.com, orcid.org/0000-0002-7841-1470

${ }^{3}$ Centro de Investigación en Matemática Educativa, Facultad de Matemáticas, Universidad Autónoma de Guerrero,gmartinez@uagro.mx, orcid.org/0000-0002-2462-7401 
algunas implicaciones prácticas y algunas recomendaciones para futuras investigaciones.

Palabras clave: creencias de profesores. valoración cognitiva. creencias profesadas. creencias implícitas

\begin{abstract}
Abstracs: Teacher beliefs research has focused on basic level teachers and teachers in training, with a great tendency to use Likert scales. On the other hand, research on teachers' emotions, from valuation theories, argues that these valuations are carried out based on beliefs, goals, etc. of the teachers. The objective of our research was to use this relationship between emotion-belief to identify, in addition to professed beliefs, beliefs implicit in the appraisal cognitive reported in daily self-reports on the class experiences of two higher-level teachers. We identified two types of implicit beliefs: referring to learning and management in the classroom. In addition, 22 mathematical beliefs professed by the teachers that we organize into five categories: mathematics is, learning mathematics is, to learn mathematics, you must teach mathematics, and to teach mathematics, you must. We present some practical implications and some recommendations for future research.
\end{abstract}

Keywords: Teachers' beliefs. Appraisal cognitive. Professed beliefs. Implicit beliefs

\title{
1. INTRODUCCIÓN
}

La investigación de creencias de profesores buscó, en sus inicios, entender el aprendizaje en las aulas desde la perspectiva de los profesores y resolver problemas de implementación de los planes y programas de estudios (Skott, 2015a; 2015b). Las creencias se consideran importantes por varias razones: (1) se piensa que las creencias guían, en gran parte, la práctica del profesor, pueden facilitar o dificultar la práctica al enmarcar y orientar sus decisiones y acciones en el aula (Skott, 2015a; Solís, 2015; Jiménez y Gutiérrez, 2017; Cross, 2015; Thompson, 1992, Philipp, 2007, Fives y Buehl, 2012); (2) se considera que las creencias de los profesores pueden moldear las creencias de los estudiantes (Schoenfeld, 1992), porque mientras aprenden matemáticas los estudiantes también están aprendiendo qué son las matemáticas, qué valor tienen, cómo se aprende, quién 
debe aprender, etc. (Skott, 2009a; Philipp, 2007), y (3) las creencias de los profesores filtran todo aquello que supone el proceso de enseñanza y aprendizaje de la matemática (García, Azcarate, y Moreno, 2006). De modo que las creencias no deben interpretarse como predictores, sino como indicadores de las intenciones que se tienen de cómo actúa un profesor (Andrews y Xenofontos, 2015).

Los puntos sobre la importancia del estudio de las creencias de profesores ponen énfasis en el papel de dichas creencias como indicadores de las decisiones que toman en el aula y en la forma en cómo se relacionan con sus estudiantes a través de sus creencias. En ese sentido, en el estudio de creencias de profesores en Matemática Educativa, se ha destacado la investigación sobre creencias acerca de las matemáticas, su enseñanza y aprendizaje -denominadas creencias matemáticas (Kul y Celik, 2017) - (Beswick, 2007; Cross, 2009; Handal, 2003; Liljedahl, 2009; Philipp, 2007; Raymond,1997; Stipek, Givvin, Salmon, y Macgyvers, 2001; Thompson, 1992; Žalská, 2012). En su conjunto, estas investigaciones muestran, que la manera en que los profesores conciben la enseñanza y el aprendizaje de las matemáticas y, en consecuencia, su práctica, se fundamenta en sus creencias acerca de la naturaleza y función de las matemáticas.

Por la importancia de éstas, se esperaría que los nuevos retos y desafíos a los que se enfrentan en sus aulas influyan en sus creencias, por tanto, la investigación de las creencias de los profesores se hace necesaria en contextos específicos y de forma recurrente. Gran parte de la investigación sobre el tema se ha centrado en profesores de nivel básico (Perry, y Howard, 1999; Grootenboer, 2008; Förster, 2011; Furinghetti y Morselli, 2011; Beswick, 2012; Misfelddt, Jankvist, y Sánchez, 2016) o en profesores en formación (Kul y Celik, 2017; Charalambous, Panaoura y Philppou, 2009; Riley, 2018; Hidalgo, Maroto, y Palacios, 2015), los cuales se ven influenciados por los planes y programas de estudios que los rigen. Consideramos importante explorar nuevos contextos en los que las creencias de los profesores juegan un papel igualmente importante, ya que, aunque los profesores de nivel superior son autónomos en sus cátedras (García, Azcárate y Moreno, 2006) sus creencias serán, con mayor razón, las guías para indicar y dirigir su actuar en el aula.

En lo que respecta a las tradiciones metodológicas, en las investigaciones de creencias de profesores ha sido común utilizar las escalas Likert. Pero las escalas Likert, por su naturaleza, se restringen a preguntar sobre el grado de acuerdo con oraciones previamente elegidas por los investigadores sin dar oportunidad a los profesores de externar con sus palabras e ideas lo que creen 
(Abd-El-Khalick y Lederman, 2000; Di Martino y Sabena, 2010). Por lo tanto, es importante considerar propuestas metodológicas que, permitan describir las creencias matemáticas de los profesores considerando métodos introspectivos y de muestreo constante de la experiencia, que puedan arrojar información más detallada que las escalas Likert y más cercanas a las experiencias particulares de los profesores, expresadas desde sus propias ideas con sus palabras.

Una propuesta metodológica son los auto-informes diarios, utilizada en Martínez-Sierra, Arellano-García, Hernández-Moreno y Nava-Guzmán (2018). El principal objetivo en Martínez-Sierra, et. al. (2018) fue estudiar las experiencias emocionales de un profesor de matemáticas de nivel medio superior en sus clases de cálculo, basados en la teoría cognitiva de las emociones (OCC). La OCC propone que las emociones son producto de la valoración cognitiva que los individuos hacen sobre situaciones que contribuyen o restan a su bienestar, existen antecedentes de valoración (metas, actitudes y creencias) con base en las que los individuos interpretan sus experiencias significativas. Martínez-Sierra, et. al. (2018) concluyeron que las creencias de cómo se aprende matemáticas son la base de la estructura de valoración cognitiva de las emociones experimentadas por el profesor. Con base en ello, plantearon la hipótesis de que las creencias matemáticas -creencias de lo que son las matemáticas, su enseñanza y su aprendizaje- son los principales antecedentes de valoración cognitiva que soportan las experiencias emocionales de los profesores en el aula. Con esta referencia, partimos de la hipótesis de que es posible identificar grupos de creencias a través del análisis de los antecedentes de valoración cognitiva de las situaciones que desencadenan una experiencia emocional de los profesores. En ese mismo sentido, Frijda y Mesquita (2000) afirman que las creencias se encuentran entre los antecedentes de valoración cognitiva de los eventos que provocan emociones de las personas y esas emociones pueden expresar creencias, a menudo, sólidas. Las creencias, entre otras "preocupaciones", es aquello en lo que se basan los individuos para detectar y evaluar la importancia del medio ambiente para el bienestar del individuo que valora un evento (Moors, Ellsworth, Scherer, y Frijda, 2013, p. 119) y que en consecuencia le desencadenan una experiencia emocional.

En la literatura de creencias de profesores se realiza una clasificación en función de cómo se identifican, creencias profesadas, professed beliefs or espoused beliefs, que se identifican por medio de lo que los profesores dicen y creencias atribuidas, attributed beliefs or enacted beliefs, que son inferidas de lo que los profesores hacen (Zhang y Morselli, 2016; Fives y Buehl, 2012; Philipp, 2007). 
Con la hipótesis planteada definimos las creencias implícitas en las valoraciones cognitivas, implicit beliefs, como aquellas que podemos identificar a través de la valoración cognitiva de las situaciones que desencadenan emociones en los profesores.

Con todo lo expuesto, el objetivo de esta investigación es identificar creencias matemáticas de profesores de nivel superior implícitas y profesadas, estudiando las valoraciones cognitivas de situaciones que desencadenan experiencias emocionales mediante auto-informes diarios, que son un método de muestro constante de la experiencia. En consecuencia, la pregunta de investigación es: ¿Cuáles son las creencias matemáticas implícitas y profesadas de profesores de matemáticas de nivel superior narradas en auto-informes diarios?

\subsection{ESTUDIOS SOBRE EMOCIONES DE PROFESORES QUE MUESTRAN SU RELACIÓN CON LAS CREENCIAS}

Para Schutz, Hong, Cross y Osbon (2006) las emociones son el resultado de "juicios conscientes y/o inconscientes con respecto a los éxitos percibidos para alcanzar las metas o mantener estándares o creencias" (p. 346). Las metas, estándares y creencias son construcciones centrales de organización de las emociones porque representan puntos de referencia utilizados por los maestros y estudiantes para determinar qué tan exitosos se ven a sí mismos en su intento por alcanzar sus objetivos. Las valoraciones son esenciales para que surjan las emociones porque "estas evaluaciones surgen de creencias y teorías personales sobre el mundo y están dirigidas a hacer comparaciones entre las metas de los individuos y dónde se perciben a sí mismas en relación con esas metas" (p. 346). Por lo que, las creencias resultan ser la base en las valoraciones de las situaciones durante las experiencias emocionales en el aula.

En ese sentido, el modelo de Frenzel (2014), propone que las emociones de los profesores resultan de los juicios que hacen con respecto al éxito o fracaso de sus propios esfuerzos de enseñanza, ya que estos parecen ser particularmente importantes. En su modelo, Frenzel especifica que los profesores evalúan cognitivamente las actividades en su salón de clases a partir de metas preestablecidas y, en función de sus juicios respecto a si las conductas de los alumnos están alineadas con ellas. Frenzel señala que para entender los antecedentes de las emociones de profesores, necesitamos identificar los ideales de enseñanza, es decir, las visiones generales de los maestros de lo que desean lograr a 
través de la instrucción e, inferir las metas resultantes que los maestros utilizan para medir sus éxitos y fracasos. Estos ideales de enseñanza tendrán distintas fuentes, entre ellas las experiencias de enseñanza recurrentes y los estándares que, desde nuestro punto de vista se consolidan en creencias sobre cómo lograr la enseñanza y el aprendizaje, así como qué son y qué significan las matemáticas, la enseñanza y el aprendizaje.

En un estudio más cercano, Martínez-Sierra, et al. (2018) mostraron que en nivel medio superior las emociones de profesores son desencadenadas principalmente por las normas de conducta esperadas de los estudiantes y las metas de clase. En donde el concepto de "actitud de los estudiantes" juega un papel importante, este concepto de actitud propio del profesor (son una serie de actividades y conductas que el estudiante muestra ante la clase, tales como la participación, la colaboración y, la autonomía) muestra que las creencias acerca de las normas, comportamientos, aprendizaje, enseñanza y naturaleza de las matemáticas son elementos sólidos de la estructura de valoración cognitiva de las situaciones que desencadenan una experiencia emocional en el profesor.

Los estudios mencionados evidencían que las experiencias emocionales de los profesores surgen a partir de las valoraciones basadas en sus creencias. Esto nos permite considerar a las creencias como los antecedentes de la valoración cognitiva de las situaciones que desencadenan una experiencia emocional en los profesores $y$, dan pie a pensar que es posible identificar creencias a través del análisis de las experiencias emocionales de los profesores.

\section{MARCO CONCEPTUAL}

\subsection{CREENCIAS Y TIPOS DE CREENCIAS}

Skott (2015a) propone cuatro aspectos fundamentales que constituyen el núcleo del concepto de creencia:

- Las creencias se utilizan generalmente para describir las construcciones mentales individuales, que son subjetivamente ciertas para la persona.

- Hay aspectos cognitivos, así como afectivos en las creencias o, por lo menos, las creencias y los problemas afectivos son vistos como ligados incomprensiblemente. 
- Las creencias se consideran, en general, reificaciones temporales y contextualmente estables, que pueden cambiar como resultado de la participación sustancial en las prácticas sociales relevantes.

- Se espera que las creencias influyan significativamente en la forma en que los profesores interpretan las situaciones para comprometerse con los problemas de la práctica.

En este trabajo se considera que el término creencia se usa para describir las construcciones mentales individuales, que son subjetivamente ciertas para los profesores en cuestión, que tienen cierto grado de convicción y no son consensuadas (Skott, 2015a), además de estar profundamente ligadas con los aspectos afectivos, tales como las emociones. Para fines operativos, consideramos la postura de Pajares (1992, p. 18) quién define una creencia como "el juicio de verdad o la falsedad de una proposición" para identificar las creencias a partir de las narraciones de los profesores.

Para este estudio, es de gran relevancia la clasificación que encontramos en la literatura sobre creencias de profesores en función de cómo se identifican: creencias profesadas (professed beliefs or espoused beliefs) como las que se identifican por medio de lo que los profesores dicen y creencias atribuidas (attributed beliefs or enacted beliefs) que son inferidas de lo que los profesores hacen (Zhang y Morselli, 2016; Fives y Buehl, 2012; Philipp, 2007). Estos dos tipos de creencias pueden ser compatibles cuando lo que se profesa se corresponde con lo que se hace en el aula o, incompatibles cuando lo que se diga no se corresponda con lo que se infiere de lo que hace.

Cuando las creencias profesadas y atribuidas son inconsistentes debe tomarse en cuenta que "las creencias no deben interpretarse como predictores, sino como indicadores de las intenciones que se tienen de cómo actuar" (Andrews y Xenofontos, 2015, p. 301) y por tanto las circunstancias o el contexto de la clase obligan al profesor a adaptar sus acciones por sobre sus creencias, además los investigadores interesados en la relación creencias y práctica, hacen la inferencia de las creencias atribuidas en contextos distintos en los que se han identificado las creencias profesadas.

En esta investigación se pretende identificar creencias desde los antecedentes de valoración cognitiva que desencadenan emociones en las experiencias diarias reportadas por los profesores. Dado que no se corresponde con las definidas en la literatura de creencias de profesores se ha decidido llamar creencias implícitas a las creencias identificadas a través del análisis de las 
valoraciones cognitivas que desencadenan emociones, debido a que estas creencias no son declaradas por el profesor ni interpretadas de sus acciones en aula, sino inferidas de las valoraciones cognitivas sobre lo que los profesores consideran importante para su bienestar en el aula.

Las teorías de valoración cognitiva de las emociones proponen que las personas experimentan emociones de acuerdo a sus valoraciones o interpretación cognitiva [appraisal] de la situación en específico. La valoración es un proceso que detecta y evalúa la importancia del medio ambiente para el "bienestar", se conceptúa como la facilitación o la obstrucción de las "preocupaciones". Por ejemplo, la ley del significado situacional de Frijda (2007) considera que "las emociones surgen en respuesta a la estructura significativa de una situación" (p. 4) por lo que la valoración implica una interacción entre el evento y quién valora el evento (Lazarus, 1991). La valoración puede ser positiva cuando la situación se evalúa como facilitadora para el bienestar del individuo o puede ser negativa cuando la situación se evalúa como una obstrucción. Así, se denomina antecedente de valoración a todas aquellas ideas que soportan las valoraciones, estas pueden ser: metas, normas, actitudes, creencias, entre otras. Nuestra investigación se centrará en identificar las creencias en la estructura de valoración de los profesores, a través de las valoraciones cognitivas de las situaciones que valoran como positivas o negativas.

\section{METODOLOGÍA}

La investigación es de tipo cualitativa y un estudio doble de caso, que nos permite una comprensión profunda de la naturaleza y la complejidad del fenómeno en estudio: las creencias matemáticas de los profesores de nivel superior (Yin, 2009). Particularmente, optamos por un estudio doble de caso instrumental (Stake, 2005) porque de un grupo de cuatro profesores de matemáticas de nivel superior que aceptaron participar, de manera voluntaria, como informantes en la investigación, escogimos a dos profesores por su interés y su disponibilidad para compartir la información necesaria en la investigación.

Yin (2009) identifica tres pasos en el diseño de estudios de casos: definir el caso, justificar la selección de un estudio de caso o un estudio de caso múltiple y, articular explícitamente cómo las perspectivas teóricas guían o deliberadamente no guían el caso. En esta investigación reportamos un estudio doble de caso instrumental -es decir; un caso en donde éste se hace para someter a 
prueba una teoría (Stake, 2005)- acerca de las creencias matemáticas (profesadas e implícitas) de dos profesores universitarios de matemáticas (GA y EDO), a través del cual intentamos avanzar en lo que se sabe sobre los diferentes tipos de creencias de profesores; en particular avanzar en el conocimiento teórico y metodológico de las creencias implícitas. Deliberadamente utilizamos las perspectivas teóricas antes detalladas para guiar nuestro diseño y análisis de este estudio.

\subsection{PARTICIPANTES}

Son dos profesores de matemáticas de nivel superior, de diferentes estados del norte de México. Uno de ellos en el momento de la toma de datos impartía el curso de Ecuaciones Diferenciales Ordinarias I y el otro un curso de Grupos y Anillos. El profesor de Ecuaciones Diferenciales Ordinarias I (en adelante profesor EDO) tiene 39 años, licenciado, maestro y doctor en matemáticas, actualmente es profesor-investigador, cuenta con 17 años de experiencia docente. El profesor de Grupos y Anillos (en adelante GA) tiene 43 años, licenciado en matemáticas, maestro en matemática educativa, maestro en matemáticas y doctor con especialidad en matemática educativa, cuenta con 17 años de experiencia docente.

\subsection{RECOLECCIÓN DE DATOS}

Consintió en una entrevista semiestructurada inicial con el objetivo de conocer aspectos generales sobre la formación de los profesores y sus opiniones respecto a la matemática, su enseñanza y aprendizaje. Aunque, la información proveniente de la entrevista semiestructurada, solo se utilizó para la descripción de los participantes, porque el interés se centró en la información recolectada de los auto-informes diarios.

Los cuales consisten en "repetidos auto-informes que tienen como objetivo capturar eventos, reflexiones, estados de ánimo, o interacciones cerca del momento en que ocurren" (lida, Shrout, Laurenceau, y Bolger, 2012, p. 277). Ofrecen, además, proximidad a la experiencia de los participantes puesto que los datos se recogen sobre su vida a medida que suceden, lo que nos permite identificar las creencias que los profesores ponen en juego durante la gestión de su clase, además reduce en gran medida el sesgo de la retrospección que 
está asociado a la encuesta habitual o diseño de la entrevista y ofrece la posibilidad de estudiar el cambio intraindividual de procesos, pensamientos, sentimientos y comportamientos en contextos muy específicos (lida et al., 2012; Zirkel, García, y Murphy, 2015). En consideración con lo anterior, se eligió el uso del

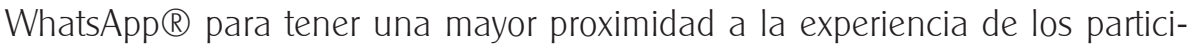
pantes. Además, de experiencias previas en el grupo de investigación con el uso del WhatsApp, que resultaron exitosas.

Los auto-informes se enviaron por este medio, minutos después de terminar cada clase y, estuvieron guiados por un protocolo (Tabla 1), con una serie de preguntas abiertas detonantes de discurso referente a lo que sucedió en el aula. En el protocolo, las preguntas 1 y 2 sirvieron de control; las preguntas 3-5 para conocer la valoración cognitiva de las situaciones en el aula y las preguntas 6-8 para conocer las creencias matemáticas profesadas por los profesores participantes y detectar experiencias significativas que pudieran propiciar algún cambio de creencias.

Tabla 1. Protocolo para los auto-informes diarios

NOTA: Debido a que solemos olvidar detalles de nuestra experiencia de manera muy rápida, recuerda que es importante contestar las siguientes preguntas minutos después de que la clase haya terminado.

Enviar en un solo audio las respuestas de todas y cada una:

1 Díganos su nombre, la fecha y hora del informe.

2 ¿De qué curso es este informe? ¿A qué hora impartió la clase?

3 ¿Cómo se sintió durante su clase? Y ¿por qué?

4 Cuéntenos las experiencias positivas que haya vivido hoy en la clase de matemáticas ¿Por qué las considera experiencias positivas?

5 Cuéntenos las experiencias negativas que haya vivido hoy en la clase de matemáticas ¿Por qué fueron experiencias negativas?

6 ¿Considera que su vivencia o experiencia de la clase de hoy cambió su idea o concepto de lo que son las matemáticas? ¿Por qué?

7 ¿Considera que su vivencia o experiencia de la clase de hoy cambió su idea o concepto de lo que es aprender matemáticas? ¿Por qué?

8 ¿Considera que su vivencia o experiencia de la clase de hoy cambió su idea o concepto de lo que es enseñar matemáticas? ¿Por qué? 
Los auto-informes se recolectaron en el periodo de septiembre a noviembre, con base en la disposición de tiempo de los profesores. Se recolectaron 9 para EDO y 21 para GA, de 5-9 minutos cada uno. La diferencia de la cantidad de auto-informes por profesor se debió a que EDO contaba con un número menor de clases a la semana (mayor cantidad de horas por sesión). Además, de algunas dificultades en su institución, por ejemplo, suspensión de clase. Mientras que GA, impartía una mayor cantidad de clase semana con menor horas por sesión.

\section{ANÁLISIS DE DATOS}

Para el análisis de los auto-informes y la presentación de los resultados, utilizamos Rn-X para cada uno, con X=EDO o GA según corresponda y n varía de 1-9 para el profesor de EDO o 1-21 para el profesor de GA.

El conjunto de datos obtenidos fue transcrito y leído repetidamente para familiarizarse con el lenguaje de los profesores. El análisis de los mismos se realizó por profesor en cuatro fases: (1) Identificación de extractos que contengan creencias profesadas o valoraciones cognitivas de situaciones; (2) Identificación de las creencias profesadas y de las creencias implícitas (3) Triangulación entre investigadores (4) Organización de las creencias en tipos de creencias y presentación de los resultados.

\section{FASE 1. IDENTIFICACIÓN DE EXTRACTOS QUE CONTENGAN CREENCIAS PROFESADAS O VALORACIONES COGNITIVAS}

En esta fase se identificaron extractos sobre creencias matemáticas, se organizaron en profesadas cuando los profesores reconocen explícitamente que es una creencia, nos guiamos por frases como "yo creo que...", "para mi...", "yo pienso que debe ser..." "tal cosa es ..., "la verdad es ..." "siempre se debe de..." "para... se tiene que...", y en creencias implícitas cuando las inferimos a través de la valoración cognitiva de las situaciones que desencadenan una experiencia emocional. Para las implícitas consideramos extractos que expresan una valoración positiva o negativa de la situación suscitada en el aula, por ejemplo: 
R1-EDO: "En cuanto a las experiencias positivas, siento que hoy pusieron un poco más atención, por lo regular están en el teléfono, están mandándose recaditos en papeles, [... y pues ahora si pusieron más atención [...]. La parte negativa es que una estudiante que por lo regular no pone mucha atención estaba tejiendo durante la clase. Estoy explicando, y ella está tejiendo ahí un suéter o no sé qué este haciendo [tono molesto]. Ok, esa la parte negativa".

En este episodio identificamos la valoración cognitiva de las situaciones que desencadenan una experiencia emocional cuando el profesor EDO hace alusión a sus experiencias positivas y negativas, valoración positiva y negativa respectivamente, en la que buscamos los antecedentes con base en lo que el profesor realiza esa valoración, para el ejemplo observamos que es importante que los estudiantes pongan atención durante la clase, por lo que se convierte en un claro candidato de ser una creencia implícita en la valoración emocional.

\section{FASE 2. IDENTIFICACIÓN DE LAS CREENCIAS PROFESADAS E IMPLÍCITAS}

Una vez separadas los extractos del discurso de los profesores se construyó una tabla (Tabla 2) por cada auto-informe para organizar los extractos y tener una visión general de las creencias de los profesores. En la Tabla 2 utilizamos de ejemplo R1-EDO.

Tabla 2. Extractos de creencias implícitas y profesadas de R1-EDO

Extractos

Creencias

\begin{tabular}{|c|c|c|c|}
\hline Valoración (+) & $\begin{array}{l}\text { En cuanto a las experiencias positivas, siento } \\
\text { que hoy pusieron un poco más atención, por lo } \\
\text { regular están en el teléfono, están mandándose } \\
\text { recaditos en papeles, [...]y pues ahora si pusie- } \\
\text { ron más atención. Algunas [estudiantes] si par- } \\
\text { ticiparon más de lo que otras veces. Y entonces } \\
\text { fue la experiencia positiva. }\end{array}$ & $\begin{array}{l}\text { Los estudiantes deben } \\
\text { poner atención en la cla- } \\
\text { se [para que logren } \\
\text { aprender matemáticas] }\end{array}$ & 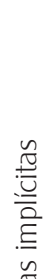 \\
\hline Valoración (-) & $\begin{array}{l}\text { La parte negativa es que una estudiante que de } \\
\text { por lo regular no pone mucha atención que di- } \\
\text { gamos estaba tejiendo durante la clase. Estoy } \\
\text { explicando, y ella está tejiendo ahí un suéter o } \\
\text { no sé qué este haciendo [tono molesto]. Ok, esa } \\
\text { la parte negativa }\end{array}$ & $\begin{array}{l}\text { Es importante que los es- } \\
\text { tudiantes participen du- } \\
\text { rante la clase. }\end{array}$ & 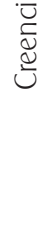 \\
\hline
\end{tabular}




\begin{tabular}{|c|c|c|c|}
\hline $\begin{array}{c}\text { Aprender } \\
\text { matemáticas }\end{array}$ & $\begin{array}{l}\text { Reafirma lo que tengo pensado [aprender mate- } \\
\text { máticas] tienen que ver con si es una clase inte- } \\
\text { resante, si al estudiante le parece interesante o } \\
\text { al menos tiene una motivación para aprender } \\
\text { l... si al menos existe una motivación para poner } \\
\text { atención, entonces, a lo mejor la clase puede } \\
\text { llevarse mejor. }\end{array}$ & $\begin{array}{l}\text { Para aprender matemáti- } \\
\text { cas la clase debe parecer- } \\
\text { le interesante a los } \\
\text { estudiantes. } \\
\text { Para aprender matemáti- } \\
\text { cas los estudiantes deben } \\
\text { tener una motivación } \\
\text { para aprender }\end{array}$ & \\
\hline $\begin{array}{c}\text { Enseñar } \\
\text { matemáticas }\end{array}$ & $\begin{array}{l}\text { En cuanto al concepto de enseñar matemáticas } \\
\text { [...] siento que se trabaja mejor si existe un am- } \\
\text { biente de colaboración tanto del profesor } \\
\text { como del estudiante, ese ambiente se logra si } \\
\text { realmente están metidos en la clase, no nada } \\
\text { más copiando lo que estoy escribiendo [...] inclu- } \\
\text { so si escribo con errores y lo copian con errores. } \\
\text { Sin realmente reflexionar si está bien o está mal } \\
\text { antes de escribir. }\end{array}$ & $\begin{array}{l}\text { Para enseñar matemáti- } \\
\text { cas debe existir un am- } \\
\text { biente de colaboración } \\
\text { profesor-estudiante. } \\
\text { Para aprender matemáti- } \\
\text { cas los estudiantes deben } \\
\text { reflexionar lo que hacen } \\
\text { no solo copiar. }\end{array}$ & \\
\hline
\end{tabular}

\section{FASE 3. TRIANGULACIÓN ENTRE INVESTIGADORES}

Consistió en reuniones regulares en las que cada uno de los investigadores presentó sus interpretaciones en el proceso del análisis de los datos y éstas fueron discutidas a fin de llegar a puntos de acuerdo sobre las creencias que cada uno identificó en los extractos de discurso. La triangulación entre investigadores permite obtener la interpretación de varios investigadores sobre un mismo conjunto de datos, de modo que se discute la pertinencia y la validez de los análisis y esto ayuda a reducir la subjetividad propia del análisis cualitativo.

\section{FASE 4. ORGANIZACIÓN DE LAS CREENCIAS EN TIPOS Y PRESENTACIÓN DE LOS RESULTADOS}

Ya identificadas las creencias, tanto implícitas como profesadas de cada uno de los auto-informes presentados por los profesores, se procedió a vaciar la información en una sola tabla (Tabla 3 y Tabla 4) para cada tipo. Para las creencias profesadas se optó por presentar solo las distintas creencias identificadas, sin tomar en cuenta la cantidad de veces que se identificó en los auto-informes. 


\section{RESULTADOS}

En la Tabla 3 se muestran las creencias implícitas en las valoraciones cognitivas y en la Tabla 4 se presentan las creencias profesadas por los profesores en los auto-informes diarios.

Tabla 3. Creencias implícitas de los profesores EDO y GA

\begin{tabular}{|c|c|c|c|c|}
\hline \multirow{2}{*}{ Creencia implícita } & \multicolumn{2}{|c|}{$\begin{array}{c}\text { EDO } \\
\text { Valoración }\end{array}$} & \multicolumn{2}{|c|}{$\begin{array}{c}\text { GA } \\
\text { Valoración }\end{array}$} \\
\hline & $f_{+}$ & $f-$ & $f+$ & $f-$ \\
\hline $\begin{array}{l}\text { Los estudiantes deben poner } \\
\text { atención en la clase [para } \\
\text { aprender matemáticas] }\end{array}$ & $\begin{array}{l}\text { R1, R3, R7 } \\
\text { (3) }\end{array}$ & $\begin{array}{l}\text { R1, R2, R6, } \\
\text { R8, R9 } \\
\text { (5) }\end{array}$ & $\begin{array}{l}\text { R1, R6, R7, R9, } \\
\text { R10, R14, R17 } \\
\text { (7) }\end{array}$ & $\begin{array}{l}\mathrm{R} 2, \mathrm{R} 12 \\
\text { (2) }\end{array}$ \\
\hline $\begin{array}{l}\text { Si los estudiantes participan, } \\
\text { aprenden porque se involucran } \\
\text { y forman parte de su propio co- } \\
\text { nocimiento }\end{array}$ & $\begin{array}{l}\text { R1, R3, R5, } \\
\text { R7, R8, R9 } \\
\text { (6) }\end{array}$ & $\begin{array}{l}\text { R3, R5 } \\
\text { (2) }\end{array}$ & $\begin{array}{l}\text { R2, R5, R10, R11, } \\
\text { R16, R19, R20, } \\
\text { R21 } \\
\text { (8) }\end{array}$ & \\
\hline $\begin{array}{l}\text { Los estudiantes deben resolver } \\
\text { problemas para aprender mate- } \\
\text { máticas }\end{array}$ & $\begin{array}{l}\text { R2, R3, R4, } \\
\text { R5, R6, R7 } \\
\text { (6) }\end{array}$ & $\begin{array}{l}\text { R5 } \\
(1)\end{array}$ & $\begin{array}{l}\mathrm{R} 8, \mathrm{R} 11, \mathrm{R} 15, \\
\text { (3) }\end{array}$ & $\begin{array}{l}\text { R3, R8, R13, } \\
\text { R17 } \\
\text { (4) }\end{array}$ \\
\hline $\begin{array}{l}\text { Si los estudiantes pasan al pi- } \\
\text { zarrón se puede interactuar con } \\
\text { ellos y se desarrolla mejor la } \\
\text { clase }\end{array}$ & $\begin{array}{l}\text { R2, R7, R9 } \\
\text { (3) }\end{array}$ & & $\begin{array}{l}\text { R2, R11 } \\
\text { (2) }\end{array}$ & \\
\hline $\begin{array}{l}\text { Los estudiantes deben mostrar } \\
\text { interés por aprender matemáticas }\end{array}$ & $\begin{array}{l}\text { R6 } \\
(1)\end{array}$ & $\begin{array}{l}\text { R3 } \\
(1)\end{array}$ & $\begin{array}{l}\mathrm{R} 1, \mathrm{R} 2, \mathrm{R} 7 \\
\text { (3) }\end{array}$ & $\begin{array}{l}\text { R15, R19 } \\
\text { (2) }\end{array}$ \\
\hline $\begin{array}{l}\text { Si los estudiantes aportan ideas } \\
\text { en las clases se trabaja mejor }\end{array}$ & & & $\begin{array}{l}\text { R2, R4, R5, R6 } \\
\text { (4) }\end{array}$ & $\begin{array}{l}\mathrm{R} 8, \mathrm{R} 11, \mathrm{R} 18 \\
\text { (3) }\end{array}$ \\
\hline Total de Valoraciones & 19 & 9 & 27 & 11 \\
\hline
\end{tabular}

Nota: $f+$ denota frecuencia de valoraciones positivas y $f$ - para valoraciones negativas. 
Tabla 4. Creencias matemáticas profesadas de EDO y GA

\section{Creencias acerca de las matemáticas}

\begin{tabular}{|c|c|c|}
\hline \multirow{2}{*}{$\begin{array}{l}\text { Matemáticas } \\
\text { es... }\end{array}$} & EDO & resolver problemas \\
\hline & GA & una forma de pensar y comprender la realidad que nos rodea \\
\hline \multicolumn{3}{|c|}{ Creencias acerca de aprender matemáticas } \\
\hline \multirow{2}{*}{$\begin{array}{l}\text { Aprender } \\
\text { matemáticas } \\
\text { es... }\end{array}$} & EDO & hacer matemáticas (resolver problemas) \\
\hline & GA & $\begin{array}{l}\text { construir razonamientos propios para resolver problemas } \\
\text { reflexionar, analizar, pensar y dar respuestas coherentes }\end{array}$ \\
\hline \multirow[t]{2}{*}{$\begin{array}{l}\text { Para aprender } \\
\text { matemáticas se } \\
\text { debe... }\end{array}$} & EDO & $\begin{array}{l}\text { tener interés y motivación de quien aprende } \\
\text { hacer problemas de forma independiente } \\
\text { aprovechar los espacios que dan los profesores para aclarar dudas } \\
\text { pensar alrededor de los conceptos } \\
\text { interactuar con el profesor }\end{array}$ \\
\hline & GA & $\begin{array}{l}\text { ser constante en la resolución de problemas } \\
\text { tener iniciativa (adelantarse en los contenidos) }\end{array}$ \\
\hline \multicolumn{3}{|r|}{ Creencias acerca de enseñar matemáticas } \\
\hline \multirow[t]{2}{*}{$\begin{array}{l}\text { Enseñar } \\
\text { matemáticas es }\end{array}$} & EDO & $\begin{array}{l}\text { propiciar la reflexión acerca del concepto que se quiere enseñar } \\
\text { poder guiar en el descubrimiento } \\
\text { realizar problemas en clase }\end{array}$ \\
\hline & GA & $\begin{array}{l}\text { inducir la abstracción de ciertas propiedades planteando preguntas } \\
\text { guías }\end{array}$ \\
\hline \multirow[t]{2}{*}{$\begin{array}{l}\text { Para enseñar } \\
\text { matemáticas se } \\
\text { debe }\end{array}$} & EDO & $\begin{array}{l}\text { propiciar un ambiente de cooperación entre profesor-estudiantes } \\
\text { tener interés y motivación de los estudiantes } \\
\text { interactuar con todos los estudiantes y pasarlos al pizarrón }\end{array}$ \\
\hline & GA & $\begin{array}{l}\text { plantear situaciones problemáticas } \\
\text { dar sólo algunas ideas y permitir que el estudiante reflexione } \\
\text { pensar y deducir enfrente de los estudiantes }\end{array}$ \\
\hline
\end{tabular}




\section{DISCUSIÓN Y CONCLUSIONES}

\subsection{RESUMEN DE LOS RESULTADOS}

Se identificaron seis creencias implícitas en las valoraciones cognitivas de los participantes, que pueden clasificarse en dos tipos: aquellas referidas al aprendizaje (poner atención, participar, resolver problemas y mostrar interés) y aquellas referidas a la gestión de clase (pasar al pizarrón y proponer ideas). Las creencias implícitas 'Los estudiantes deben poner atención en la clase Ipara aprender matemáticas]' y 'Si los estudiantes participan, aprenden porque se involucran y forman parte de su propio conocimiento' son las que más valoraciones cognitivas emocionales desencadenaron en ambos profesores. Las creencias implícitas son compartidas exceptuando 'Si los estudiantes aportan ideas en las clases se trabaja mejor', que sólo se identificó en el profesor GA.

Se identificaron 22 creencias matemáticas profesadas por los profesores del estudio, que organizamos en cinco categorías (Matemáticas, aprender matemáticas, para aprender matemáticas, enseñar matemáticas y para enseñar matemáticas). Para GA las matemáticas son "una forma de pensar y comprender la realidad" por tanto, "para aprender matemáticas el estudiante debe ser capaz de reflexionar, analizar, pensar y construir razonamientos coherentes ante la resolución de problemas", y esta creencia permea en su visión de la enseñanza en donde busca "inducir abstracciones", es importante plantear situaciones problemáticas, dar algunas ideas y permitir la autonomía de los estudiantes. Mientras que, para EDO, matemáticas es "resolver problemas", por lo que para él aprender matemáticas se debe "hacer matemáticas", lo que involucra realizar problemas constantemente, de manera independiente, con interés y motivación, así, el papel del profesor es guiar a los estudiantes a la reflexión y al descubrimiento a través de una interacción en el aula.

\subsection{SOBRE LAS CREENCIAS IMPLÍCITAS}

Las creencias implícitas en las valoraciones cognitivas pueden servir como una lente a través de la cual los profesores participantes observan el comportamiento de los estudiantes. Por ejemplo, en la creencia implícita 'Si los estudiantes participan, aprenden porque se involucran y forman parte de su propio conocimiento' podemos inferir que el grupo de GA, desde la perspectiva de 
GA, participó en las clases, ya que GA realizó más valoraciones cognitivas de las situaciones, evaluándolas como positivas bajo esa creencia. Mientras que el grupo de EDO parece, desde la perspectiva de EDO, más dispuesto a resolver problemas en la clase.

En las creencias implícitas destaca la importancia del aprendizaje de los estudiantes en las valoraciones cognitivas emocionales de los profesores, que se relacionan con su bienestar psicológico. Lo que hace a las creencias implícitas, creencias operativas y contextualizadas, con las que los profesores reconocen emocionalmente sus éxitos o fracasos en el salón de clases. Este resultado es consistente con lo que encontraron Martínez-Sierra et al. (2018) y Frenzel (2014), quienes señalan que la principal fuente de valoración cognitiva de las emociones de los profesores en el aula son los estudiantes y el cómo se espera que aprendan. Así como también corrobora nuestro planteamiento inicial: la importancia de las creencias matemáticas, en específico sobre el aprendizaje, son el principal antecedente de valoración emocional de los profesores.

Las creencias implícitas referentes a la participación y poner atención para aprender matemáticas son en las que más valoraciones realizaron los profesores participantes. Desde las teorías de valoración cognitiva de las emociones, esto implica que estas creencias, entre otras "preocupaciones", son en las que se basan los individuos para detectar y evaluar la importancia del medio ambiente para su bienestar (Moors, Ellsworth, Scherer, y Frijda, 2013, p. 119). Lo cual significa que, participación y atención de los estudiantes son aspectos importantes para el bienestar de los profesores en el desarrollo de la clase y, por tanto, variables a considerar en las planeaciones. Y debido a que las creencias pueden facilitar o dificultar la práctica, al enmarcar y guiar las decisiones y acciones de los profesores en el aula (Skott, 2009a, Skott, 2015; Solís, 2015; Jiménez y Gutiérrez, 2017; Cross, 2015; Thompson, 1992, Phillip, 2007, Fives y Buehl, 2012), las reacciones de los estudiantes, principalmente participary poner atención, pueden explicar algunas decisiones de los profesores participantes, por ejemplo pasar al pizarrón a algunos de los estudiantes y resolver problemas durante la clase.

\subsection{SOBRE LAS CREENCIAS PROFESADAS}

Si se considera que las creencias de los profesores pueden ser transferidas a sus estudiantes, mientras aprenden matemáticas (Skott, 2009a; Phillip, 2007), los estudiantes de EDO, estarán expuestos a creencias como que las matemáticas 
son resolver problemas, mientras que los estudiantes de GA, estarán expuestos a la creencia de que las matemáticas son una forma de pensar. Esto debido a que, EDO muestra coherencia en cuanto a que la resolución de problemas permite entender las creencias sobre lo que son las matemáticas, cómo se aprende y cómo se enseña matemáticas, el caso de GA es semejante respecto a que la naturaleza de las matemáticas es una forma de pensar, esa creencia se muestra ligada a cómo se enseña y cómo se aprenden las matemáticas. Los estudiantes estarán, a lo largo de su vida, enfrentándose a distintas visiones de las matemáticas a través de sus profesores. En este estudio identificamos dos visiones distintas, pero cada una correspondiente con la formación de los docentes.

De las creencias profesadas sobre lo que son las matemáticas para cada participante, si se considera que las creencias filtran todo aquello que supone el proceso de enseñanza y aprendizaje de la matemática (García, Azcarate, y Moreno, 2006), podemos decir que, las creencias profesadas pueden depender del curso y contexto en el que se ponen en juego, mientras que las creencias implícitas se pueden considerar un tanto independientes del contexto y del curso. Las creencias profesadas, en cambio, aunque siguen cierta coherencia entre las distintas creencias matemáticas, hacen posible que los profesores varíen su discurso para hacer adecuaciones de qué y cómo presentar el contenido, y de cómo identificar el aprendizaje de sus estudiantes en cada curso. Mientras que las valoraciones cognitivas de situaciones se realizan soportadas en creencias del comportamiento de los estudiantes, que son semejantes en cada curso.

\subsection{SOBRE EL MÉTODO DE AUTO-INFORMES}

El método de muestreo constante, auto-informes diarios, nos permitió obtener datos contextualizados y cercanos a las valoraciones cognitivas emocionales. Una de las ventajas que nos brindó es observar la consistencia y estabilidad de ciertas creencias implícitas y profesadas. Al igual que lida et al. (2012) y Zirkel, García, Murphy (2015) estamos convencidos de que el uso de los auto-informes permite el estudio de la subjetividad de las personas en contextos específicos, en este caso el aula de nivel superior, de una manera económica y ecológica, en este caso, nos permitieron analizar las creencias implícitas y profesadas de los participantes.

Si bien, el método de auto-informes diarios no había sido utilizado para identificar las creencias de los profesores durante la clase, resultó ser un medio 
novedoso para la identificación de creencias implícitas y profesadas por los profesores de este estudio. Nos permitieron conocer las creencias desde el punto de vista del profesor acerca del ambiente que vive en el aula día a día, dando indicios de su bienestar psicológico durante cada clase, puesto que cada auto informe fungió como un medio de desahogo y reflexión sobre su quehacer profesional.

\subsection{LIMITACIONES Y FUTURAS INVESTIGACIONES}

Si bien nuestra investigación no busco explícitamente alcanzar los elementos de rigor canónicos propios de una investigación cualitativa -e.g. credibilidad, auditabilidad (confirmabilidad) y transferibilidad (Denzin y Lincoln, 1994)- consideramos que debido al tipo de datos recolectados y a la triangulación de las interpretaciones de los autores, alcanzamos en cierta medida tales elementos. Si bien no presentamos los resultados con los participantes, consideramos que nuestros resultados son creíbles dado que son producto de la triangulación de interpretación de los tres autores del presente artículo y dada la consistencia entre lo que los profesores dicen creer y lo que nosotros inferimos como sus creencias implícitas. Consideramos que la investigación es auditable, debido a que aquí hemos expuesto con detalle todo nuestro proceder metodológico de recolección y análisis de datos. Si bien los resultados que encontramos no pueden ser generalizados para otros contextos, consideramos que la investigación es transferible en el sentido que en cualquier contexto es posible inferir creencias implícitas y profesadas de profesores a través de los auto-informes de las experiencias (positivas/negativas) de sus clases.

Nuestro estudio enfrenta las limitaciones propias de los análisis cualitativos, narrativos y de caso. La manera en la que accedemos a las creencias profesadas de los profesores sigue siendo a través de sus declaraciones y, aunque las creencias implícitas fueron identificadas a través de las valoraciones emocionales, estas valoraciones son extraídas de las narraciones de los profesores. Sin embargo, consideramos que debemos acceder a las creencias de los profesores desde sus propias perspectivas y valoraciones, porque a pesar de que las creencias son subjetivas, sólo podemos llegar a ellas a través de sus expresiones y es importante no anteponerles valores externos.

Futuras investigaciones pueden explorar más sobre las creencias de profesores de nivel superior, ya que el campo necesita ser desarrollado, pensamos 
que las creencias de los profesores de nivel superior podrían impactar con mayor énfasis (que de otros niveles formativos) a las creencias de los estudiantes en proceso de profesionalización.

Este estudio da cuenta de la importancia de las creencias matemáticas en las valoraciones cognitivas y el bienestar de los profesores participantes, en este caso de nivel superior, futuras investigaciones pueden profundizar en el papel de estas creencias y de los distintos tipos de creencias en las decisiones de clase que los profesores toman durante el desarrollo de su práctica.

\section{AGRADECIMIENTOS}

Agradecemos a CONACYT por la beca otorgada para llevar acabo esta investigación. Así, como a los profesores participantes y a los revisores de la revista Educación Matemática por sus atinadas observaciones que nos ayudaron para la presentación de nuestra investigación.

\section{REFERENCIAS}

Abd-El-Khalick y Lederman, N. (2000). Improving science teachers' conceptions of nature of science: a critical review of the literature. International Journal of Science Education, 22 (7), 665-701. https://doi.org/10.1080/09500690050044044

Andrew, P. y Xenofontos, C. (2015). Analysing the relationship between the problem-solving-related beliefs, competence and teaching of the three Cypriot primary teachers. Journal of Mathematics Teacher Education, 18(4), 299-325.

Beswick, K. (2007). "Teachers' beliefs that matter in secondary mathematics class- rooms". Educational Studies in Mathematics, 65(1), 95-120.

Beswick, K. (2012). Teachers' beliefs about school mathematics and mathematicians' mathematics and their relationship to practice. Educational Studies in Mathematics, 79, 127-147. https://doi.org/10.1007/s10649-011-9333-2

Charalambous, C., Panaoura, A., y Philippou, G. (2009). Using the history of mathematics to induce changes in preservice teachers'beliefs and attitudes: insights from evaluating a teacher education program, Educational Studies in Mathematics, 71, 161-180. https://doi.org/10.1007/s10649-008-9170-0 
Cross, D. I. (2009). Alignment, cohesion, and change: Examining mathematics teachers' belief structures and their influence on instructional practices. Journal of Mathematics Teacher Education, 12(5), 325-346.

Cross, D. I. (2015). Despelling the notion of inconsistencies in teachers' mathematics beliefs and practices: A 3-year case study. Journal of Mathematics Teacher Education, 18, 173-201.

Denzin, N.K. y Lincoln Y.S. (1994). Handbook of qualitative research. Sage Thousand Oaks. Di Martino, P. y Sabena, C. (2010). Teachers' beliefs: The problem of inconsistency with practice. In M. Pinto, y T. Kawasaki (Eds.). Proceedings of the 34th Conference of the International Group for the Psychology of Mathematics Education (Vol. 2), PME.

Fives, H. y Buehl, M. M. (2012). Spring cleaning for the "messy" construct of teachers' beliefs: what are they? Which have been examined? What can they tell us? In K.R. Harris, S. Graham, y T. Urdan (Eds.). APA Educational Psychology Handbook. Individual Differences and Cultural and Contextual Factors (Vol. 2, pp. 471-499). American Psychological Association. https://doi.org/10.1037/13274-019

Förster, F. (2011). Secondary Teachers' beliefs about teaching applications-Design and Selected results of a qualitative case study, In G. Kaiser et al. (Eds.), Trends in Teaching and Learning of Mathematical Modelling, International Perspectives on the Teaching and Learning of Mathematical Modelling, Springer Science+Business Media B.V. (pp. 65-74). https://doi.org/10.1007/978-94-007-0910-2_8

Frenzel, A. C. (2014). Teacher emotions. In R. Pekrun y L. Linnenbrink-Garcia (Eds.), International handbook of emotions in education (pp. 494-519). Routledge.

Frijda, N. H. (2007). The laws of emotion. Lawrence Erlbaum.

Frijda, N.; Mesquita, B. (2000). Beliefs through emotions. In N. Frijda, A. Manstead, y S. Bem (Eds.), Emotions and Beliefs: How Feelings Influence Thoughts (Studies in Emotion and Social Interaction) (pp. 45-77). Cambridge University Press. https://doi. org/10.1017/CBO9780511659904.003

Furinghetti, F. y Morselli, F. (2011). Beliefs and beyond: Hows and whys in the teaching of proof. ZDM Mathematics Education, 43, 587-599.

García, L., Azcárate, C., Moreno M. (2006). Creencias, concepciones y conocimiento profesional de profesores que enseñan cálculo diferencial a estudiantes de ciencias económicas. RELIME, 9(1), 85-116.

Grootenboer, P. (2008). Mathematical belief change in prospective primary teachers. Journal Mathematics Teacher Education, 11, 479-497. https://doi.org/10.1007/s10857-008-9084-x Handal, B. (2003). Teachers' Mathematical Beliefs: A Review. The Mathematics Educator, 13(2), 47-57. 
Hidalgo, S. Maroto, A., y Palacios, A. (2015). Una aproximación al sistema de creencias matemáticas en futuros maestros. Educación Matemática, 27(1), 65- 90.

lida, M., Shrout, P., Laurenceau, J., y Bolger, N. (2012). Using diary methods in psychological research. APA Handbook of Research Methods in Psychology. Foundations, Planning, Measures and Psychometrics, (Vol. 1, pp. 277-305). http://doi.org/10.1037/13619-016

Jiménez, A., Gutiérrez, A. (2007). Realidades escolares en las clases de matemáticas. Artículos de investigación, 29(3), 109-129. https://doi.org/10.24844/EM2903.04

Kul, U., Celik, S. (2007). Exploration of pre-service teachers' beliefs in relation to mathematics teaching activities in classroom-based setting. International Journal of Research in Education and Science (IJRES), 3(1), 245-257.

Liljedahl, P. (2009). Teachers' insights into the relation ship between beliefs and practice. En J. Maasz y W. Schlöglmann (Eds.), Beliefs and attitudes in mathematics education: New research results (pp. 44-54). Sense Publishers.

Martínez-Sierra, G., Arellano-García, Y., Hernández-Moreno, A., Nava-Guzmán, C. (2018). Daily emotional experiences of a high school mathematics teacher in the classroom: a qualitative experience-sampling method. International Journal of Science and Mathematics Education, 17(3), 1-21.

Misfelddt, M., Jankvist, U.T. y Sánchez, M.S. (2016). Teachers' beliefs about the discipline of mathematics and the use of technology in the classroom, Mathematics education, 11(2), 395-419. https://doi.org/10.12973/iser.2016.2113음

Moors, A., Ellsworth, P. C., Scherer. K. R., y Frijda, N. H. (2013). Appraisal Theories of emotion: State of the Art and Future Development. Emotion Review, 5(2), 119-124.

Perry, B., y Howard, P. (1999). Head mathematics teachers' beliefs about the learning and teaching of mathematics, Mathematics Education Journal, 11(1), 39-53.

Philipp, R. A. (2007). Mathematics teachers' beliefs and affect. In F. Lester (Ed.). Handbook of research on mathematics teaching and learning (pp. 257-315). Information Age Publishing.

Raymond, A. M. (1997). Inconsistency between a beginning elementary school teacher's mathematics beliefs and teaching practice. Journal for Research in Mathematics Education, 28(5), 550-576.

Riley, M. E. L. (2007). A typological analysis: understanding pre-service teacher beliefs and how they are transformed, International Journal of Mathematical Education in Science and Technology, 49(3), 355-383. https://doi.org/10.1080/0020739X.2017.1360526

Schoenfeld, A. H. (1992). Learning to think mathematically: Problem solving, metacognition, and sense-making in mathematics. En D. Grouws (Ed.). Handbook for Research on Mathematics Teaching and Larning (pp. 334-370). Macmillan. 
Skott, J. (2015a). The promises, problems, and prospects of research on teachers' beliefs. En H. Fives y M. G. Gill (Eds.), International Handbook of research on teachers' beliefs (pp. 13-30). Routledge.

Skott, J. (2015b). Towards a Participatory Approach to "Beliefs" in Mathematics Education. En B. Pepin y B. Roesken-Winter (Eds.), From beliefs to dynamic affect systems in mathematics education (pp. 3-23).

Skott, J. (2009a). Contextualising the notion of 'belief enactment'. Journal of Mathematics Teacher Education, 12(1), 27-46.

Solís, C. (2005). Creencias sobre la enseñanza y aprendizaje en docentes universitarios: revisión de algunos estudios. Propósitos y Representaciones, 3(2), 227-260.

Stake, R. E. (2005). Investigación con estudio de casos. Morata.

Stipek, D. J., Givvin, K. B., Salmon, J. M., y Macgyvers, V. L. (2001). Teachers' beliefs and practices related to mathematics instruction. Teaching and Teacher Education, 17(2), 213-226.

Schutz, P., Hong, J. Y., Cross, D., y Osbon, J. N. (2006). Reflections on investigating emotion in educational activity settings. Educational Psychology Review, 18(4), 343-360. https://doi.org/10.1007/s10648-006-9030-3

Thompson, A. (1992). "Teachers' beliefs and conceptions: A synthesis of the research". En D. A. Grouws (Ed.). Handbook of research on mathematics teaching and learning (pp. 127-146). Macmillan.

Yin, R. (2009). Case study research. Sage Publications.

Žalská, J. (2012). Mathematics teachers' mathematical beliefs: A comprehensive review of international research. Scientia in Educatione, 3(1), 45-65.

Zhang, Q., y Morselli, F. (2016). Summary and looking ahead. In M. S. Hannula, P. Di Martino, M. Pantziara, Q. Zhang, F. Morselli, E. Heyd-Metzuyanim, S. Lutovac, R. Kaasila, J. A. Middleton, A. Jansen y G. Goldin (Eds), Attitudes, Beliefs, Motivation and Identity in Mathematics Education: An ICME study 13 (pp. 23-26). Springer.

Zirkel, S., García, J. A., y Murphy, M. C. (2016). Experience-sampling research methods and their potential for education research. Educational Researcher, 44(1), 7-16. https://doi. org/10.3102/0013189 X14566879.

ANTONIA HERNÁNDEZ MORENO

Dirección: Centro de Investigación en Matemática Educativa-Facultad de Matemáticas-UAGRO, Av. Lázaro Cárdenas S/N, Ciudad Universitaria C.P. 39074, Chilpancingo de los Bravo, Guerrero, México

Teléfono: $\quad$ 747-77-19310 Ext. 4139 